\title{
Pathogenesis of ankylosis and re-ankylosis: the story
}

Keywords: stiff joint, ankylosis, physiotherapy, cytotoxic medication, pathogenesis, osteoblasts

Abbreviations: TMJ, temporo mandibular joint; BMP, bone morphogenic proteins; TGF-B, transforming growth factor-B

\section{Editorial}

Ankylosis is the Greek for "stiff joint". The term "ankylosis of the temporomandibular joint" (TMJ) refers to bony or fibrous adhesion of the anatomical components of the joint and their ensuing loss of function. A progressive reduction in jaw movement is the main clinical presentation. TMJ ankylosis not only affects mouth opening but also the normal growth pattern of the mandible. Outcome of surgery for TMJ ankylosis is unpredictable. The type of ankylosis and patient age should be evaluated when planning surgery. Care in the choice of surgical technique and of interposition material and subsequent meticulous attention to long-term physiotherapy are considered essential to achieve a satisfactory result. Iatrogenic causes of ankylosis may arise from cytotoxic medication, repeated TMJ surgery and irradiation. Repeated TMJ surgery emerged as a dilemma largely confined to Western countries following the introduction of the concept of internal derangement and disk displacement which acted as the catalyst for unmitigated surgical intervention in TMJ disorders during the 1970s and 1980s. ${ }^{1}$ While there have been many publications regarding the condition and its treatment, very little as been written about its pathogenesis. Due to the difficulty of operation to release the ankylosis and the high incidence in the recurrence rate, surgical treatment of bony ankylosis is still a considerable challenge for maxillofacial surgeons. Understanding the story of pathogenesis of the ankylosis and re-ankylosis might help in improving the outcome of the surgical treatment. ${ }^{2}$

Inciting event (Trauma) leads to three main events; 1- disc displacement with severe injury to the articular surfaces with formation of intra-articular hematoma rich in osteo progenitor cells (peri vascular undifferentiated pluri potential mesenchymal cells-PUPMC). 2-Change in local tissue environment includes disturbances involving the microvasculature and changes in oxygen tension, $\mathrm{pH}$ (Alkaline) and blood flow. These local changes of the tissue produce a milieu that supports osteogenesis. 3-Trauma signal lead to local release of osteo inductive cytokines especially bone morphogenic proteins (BMP), members of the transforming growth factor-B (TGF-B) super family, and genes that synthesize osteoid and chondroid (matrix) are activated. These lead to differentiation of the PUPMC into osteoblasts or chondroblasts start lay down of new bone. Trauma is thought to disrupt the normal balance of connective tissue modeling and remodeling, possibly by inducing local release of a wide array of inflammatory mediators that ultimately lead to the induction of osteogenesis. With decrease range of movement and due to lack of function after complete ankylosis, heterotrophic bone is often diffuse and does not always follow anatomic planes of tissue, which lead to excessive bone formation and formation of the ankylotic mass. ${ }^{3,4}$ Muscular atrophy regularly occurs as a consequence of immobilization. Strength decreases most dramatically during the

\author{
Volume 2 Issue 5 - 2015
}

\author{
Ayman Hegab \\ Department of Oral \& Maxillofacial Surgery,Al-Azhar University, \\ Egypt
}

Correspondence: Ayman Hegab, Clinical Associate Professor of Oral \& Maxillofacial Surgery, Al-Azhar University, Cairo, Egypt, Tel 00974 33310124,0020100198III2,

Email hegab@gmail.com, prof.aymanhegab@yahoo.com

Received: June 23,2015 | Published: June 29, 2015

first week of immobilization; little further weakening occurs later on. Long standing ankylosis associated with changes of the temporalis and master mules varied from slight degeneration of muscle fibers, to moderate or severe changes of muscle atrophy and necrosis. ${ }^{5}$ Surgical treatment of the ankylosis resulting in three major proceedings; 1creation of gap between two raw bony surfaces in close approximation especially by the action of the atrophied muscles; 2- Osseous particles and bone dust created during the surgery as a consequence of surgical cutting of bone in preparation for arthroplasty, are implanted, albeit unintentionally, into surrounding soft tissues. 3- Postoperative hematoma rich in the "Wandering histiocytes" or inductor cells. These bone matrix particles (Osseous particles and bone dust) contain osteogenic cytokines (in particular BMPs) and other growth factors that stimulate PUPMC resident in the surrounding soft tissues to differentiate into osteoblasts. ${ }^{4}$ Simultaneously with the "Wandering histiocytes" or inductor cells which activate peri vascular connective tissue cells or "fixed histiocytes" (induced cells) to differentiate into specialized osteo progenitor cells or chondro progenitor cells. The osteo progenitor cells and the stimulated BMP create the correct environment for bone formation. The progenitor cells start to form bone or cartilage that is subsequently mineralized into mature bone. Where bone induction took place, a pool of responsive cells surrounded by abundant capillaries and advancing fronts of osteoblasts were also present. ${ }^{3}$ Osteogenic cell differentiation has been shown to start within 16 hours post-operatively and peaks at 32 hours. ${ }^{6}$ In association of the contributing factors of close approximation of the articular components, and decreased joint mobility resulting from the action of the atrophied muscles lead to re-ankylosis.

\section{Acknowledgments}

None

\section{Conflicts of interest}

The author declares that there are no conflicts of interest.

\section{Funding}

None. 


\section{References}

1. Dolwick MF, Dimitroulis G. A re-evaluation of the importance of disc position in temporomandibular disorders. Aust Dent J. 1996;41(3):184187.

2. Ayman F Hegab. Outcome of surgical protocol for treatment of temporomandibular joint ankylosis based on the pathogenesis of ankylosis and re-ankylosis. A prospective clinical study of 14 patients. Journal of Oral and Maxillofacial Surgery. 2015.

3. Vanden Bossche L, Vanderstraeten G. Heterotopic ossification: a review. J Rehabil Med. 2005;37(3):129-136.
4. Lounev VY, Ramachandran R, Wosczyna MN,et al. Identification of progenitor cells that contribute to heterotopic skeletogenesis. J Bone Joint Surg Am. 2009;91(3):652-663.

5. El-Labban NG, Harris M, Hopper C, et al. Degenerative changes in masseter and temporalis muscles in limited mouth opening and TMJ ankylosis. J Oral Pathol Med. 1990;19(9):423-425.

6. Craven PL, Urist MR. Osteogenesis by radioisotope labelled cell population in implants of bone matrix under the influence of ionizing radiation. Clin Orthop Relat Res. 1971;76:231-243. 